\title{
Design and Experiment of a Conformal Monopulse Antenna for Passive Radar Applications
}

\author{
Song Lizhong ${ }^{1,2}$ \\ ${ }^{1}$ State Key Laboratory of Millimeter Waves, School of Information Science and \\ Engineering, Southeast University, Nanjing, 210096, P. R. China \\ ${ }^{2}$ School of Information and Electrical Engineering, Harbin Institute of \\ Technology at Weihai, Weihai, 264209, P. R. China \\ E-mail:songlizhong@hitwh.edu.cn
}

\begin{abstract}
This paper proposed a design scheme of wide band monopulse antenna system for the passive radar seeker (PRS) of anti-radiation missile (ARM). To save the installation space of the PRS, the conical conformal log periodic antennas (LPDA) are employed to form the monopulse antenna system. The specific implementation scheme of PRS based on the conically conformal monopulse antennas was designed and analyzed. A practical monopulse antenna system composed of two log periodic antennas was designed and fabricated over the operating frequency range of $1 \mathrm{GHz}$ to $8 \mathrm{GHz}$. A loaded resistor with impedance equal to $50 \Omega$ is used to reduce the return loss at low frequency. The experimental results demonstrate that acceptable wide band impedance matching and port isolation for each fabricated antenna was achieved. Furthermore, the wide band sum and difference radiation patterns were formed, which validates the proposed conformal monopulse antenna design scheme for the PRS application.
\end{abstract}

Keywords: passive radar, monopulse antenna, wide band antenna, conformal antenna, polarization

\section{Introduction}

The guided missiles are the main weapons in modern wars. In modern electronic warfare, the ARM is a kind of important method to destroy the radars and other radiation sources of the enemy. The PRS is the main guidance method of ARM, so there are increasing demands for the passive radars in modern military fields. In order to accurately measure the direction of the arrival of the incident electromagnetic wave, the monopulse angle measurement technique is usually used in passive radars. Monopulse radar systems require antennas able to generate sum and difference patterns to track the target accurately [1-2]. The sum pattern is used in both the signal transmission and reception to detect the target, and the difference pattern provides information on the angular position of the target. To obtain the electromagnetic wave information radiated by the radars and the other radiation sources, the passive radar should have broad operating bandwidth. The traditional band wide antennas include equiangular spiral antenna, Archimedean spiral antenna, sinuous antenna, tapered slot antenna, LPDA and so on [3-4]. The equiangular spiral antenna and Archimedean spiral antenna has the radiation characteristics of circular polarization and broad beam width, and the sinuous antenna with four arms can operate at dual polarized mode [5-6]. Another alternative 
wide band antenna with good directivity and high efficiency is the log-periodic antenna [7]. It is well known the PLDA has the periodic structure which results in constant radiation performances over ultra wide operating band. At the same time, the LPDA is convenient to manufacture due to its simple and compact structure. Until now, the LPDA is an attractive wide band antenna and was researched by many scientist and engineers. For the passive radars on the aircraft, the antenna installation spaces are usually limited, which constrain the operating frequency range. A novel method to solve this problem is to employ the conformal antenna technique [8-9]. The conformal antennas were extensively researched and often were used to the estimation of direction of arrival (DOA) of the target in radar and communication systems in recent years [1011]. The most important advantage of conformal antenna is that it can save the save the installation space and not affect the aerodynamic performances. In some cases, the beam coverage range can be enlarged and the gain can be improved for the conformal antenna. However, the antenna design becomes complicated due to its complex curved structure and installation space environment. In this paper, a design scheme of wide band monopulse antenna system employing conical conformal log-periodic antennas for passive radar is proposed. The proposed antennas can be installed on the surface of radar radome and the sum and difference patterns can be formed. The conical conformal LPDA was designed and fabricated over the frequency range of $1 \mathrm{GHz}$ to $8 \mathrm{GHz}$. A practical monopulse antenna system on the surface of a foam cone was measured to investigate the radiation performances of conical conformal monopulse antenna system. The experimental results are provided in this paper, which demonstrate that the proposed monopulse antenna system is an effective scheme for wide band passive radars.

\section{Implementation Scheme of Passive Radar Seeker based on the Conformal LPDA}

The monopulse angle measurement techniques are widely used in modern radar seekers because of their good abilities of real time processing and anti-jamming [1214]. For the monopulse angle measurement technique, the direction information of targets can be obtained by comparing the simultaneously received signals of two or multiple antennas. In theory, the two dimension angle information can be obtained from one pulse signal of radar echo for the monopulse radar seeker, and the effect of pulse echo amplitude on the estimated angle error signal can also be eliminated effectively. The sum and difference monopulse angle measurement method is the most widely used monopulse technique in engineering because it permits that the receiving channels have not strict balanced characteristics for the monopulse radar system. The passive radar seeker (PRS) of ARM usually adopts the monopulse angle measurement technique to obtain the direction information of enemy radar or other radiation sources. For the traditional PRS, the wide band monopulse antenna systems such as the planar equiangular antennas are employed, which are mounted in the antenna radome of radar and occupy large missile space. In this paper, we propose a new implementation scheme of PRS based on conformal LPDA. Compared with other antenna system of PRS, the proposed monopulse antenna can effectively save the internal space of the missile. Furthermore, the antenna sizes can be enlarged when they are mounted on the surface of the conic radome, so the good radiation performances at low frequency can be obtained and the operating band width can also be expanded. In this section, a monopulse radar system with a single plane angle measurement function was designed and analyzed to 
illustrate the proposed radar scheme. The block diagram of implementation scheme of the proposed PRS is shown Fig.1. In Fig.1, the digital monopulse technique is employed. A and B denote two ultra wide band conformal LPDAs, respectively. The signals of radiation sources are received by two antennas and are transformed to low frequency signal through two wide band microwave receivers. After Analog to Digital Converter $(A / D)$, the received signals of radiation sources are input into the digital signal processor (DSP). The target detection, target identification and angle measurement are finished in the DSP. The measured angle information of radiation source is used to guide the missile to track and destroy the radiation source.

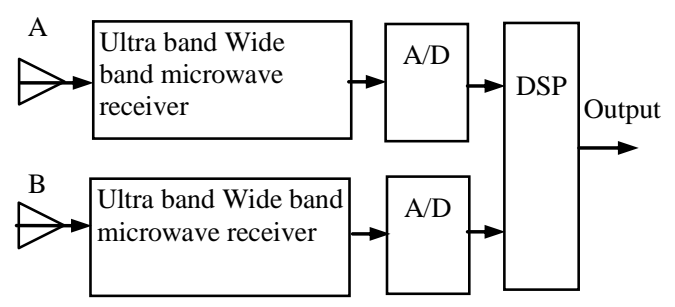

\section{Figure 1. Implementation Scheme of PRS based on Conformal Monopulse Antenna}

Generally, the antenna radome of PRS is a cone, so the wide band LPDA antennas are suitable to be mounted on the surface of the radome and the conically conformal wide band antennas can be formed. The monopulse angle measurement theorem based on the conically conformal antennas can be shown in Fig.2. In Fig.2, A and B denote two conically conformal LPDAs, and they are symmetrically mounted on the surface of a conic radome. The antenna alignment mode can achieve several favorable radiation performances for conformal monopulse antennas. The received signals of A and B can be expressed as:

$$
\dot{E}_{A}=\dot{E}_{m A} F_{A}(\theta, \varphi)\left(\mathbf{p}_{A} \cdot \mathbf{p}_{i}\right) e^{-j \frac{k d \sin \theta}{2}}
$$

$$
\dot{E}_{B}=\dot{E}_{m B} F_{B}(\theta, \varphi)\left(\mathbf{p}_{B} \cdot \mathbf{p}_{i}\right) e^{j \frac{k d \sin \theta}{2}}
$$

Where $\dot{E}_{m A}=E_{m A} e^{j \psi_{0}}$ is the signal amplitude of the antenna A, and $\dot{E}_{m B}=E_{m B} e^{j \psi_{0}}$ is the signal amplitude of the antenna B. $F_{A}(\theta, \varphi)$ and $F_{B}(\theta, \varphi)$ are the normalized pattern function of $\mathrm{A}$ and $\mathrm{B}$, respectively. $k$ is the wave number which is equal to $2 \pi / \lambda$, where $\lambda$ is the working wave length. $\theta$ and $\varphi$ are the coordinate variables in the spherical coordinate system. $\mathbf{p}_{i}$ denotes the polarization characteristic of the incident radiation source signal and it can be expressed as:

(3)

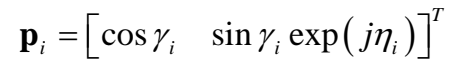

Where $\gamma_{i}$ and $\eta_{i}$ are the amplitude and phase polarization parameters of the incident signal, respectively. $\mathbf{p}_{A}$ and $\mathbf{p}_{B}$ denote the polarization characteristics of $\mathrm{A}$ and $\mathrm{B}$, respectively. In the main beam for the antennas with broad beam widths and symmetrical alignment, $\mathbf{p}_{A}$ and $\mathbf{p}_{B}$ are approximately the same, namely, $\mathbf{p}_{A}=\mathbf{p}_{B}=\mathbf{p}$, and $\mathbf{p}$ can be expressed as: 


$$
\mathbf{p}=\left[\begin{array}{ll}
\cos \gamma & \sin \gamma \exp (j \eta)
\end{array}\right]^{T}
$$

(4)

Where $\gamma$ and $\eta$ are the amplitude and phase polarization parameters of the incident signal, respectively. For the same reason, in the main beam for the monopulse antennas in this case, $\dot{E}_{m A}=\dot{E}_{m B}$ and $F_{A}(\theta, \varphi)=F_{B}(\theta, \varphi)$. So the sum signal and difference signal can be expressed as:

$$
\begin{aligned}
& \Delta=\dot{E}_{A}-\dot{E}_{B} \\
& \Sigma=\dot{E}_{A}+\dot{E}_{B}
\end{aligned}
$$

Then the monopulse ratio is obtained and it can be expressed as:

$$
\Delta / \Sigma=-j \tan (k d \sin \theta / 2)
$$

(7)

The angle error function of the monopulse antenna system can be written as:

$$
S(\theta)=\operatorname{Re}(j \Delta / \Sigma)=\tan (k d \sin \theta / 2)
$$

The angle information of radiation source can be measured according to the formula (8) and the angle measurement procedure is finished in the DSP through digital method.

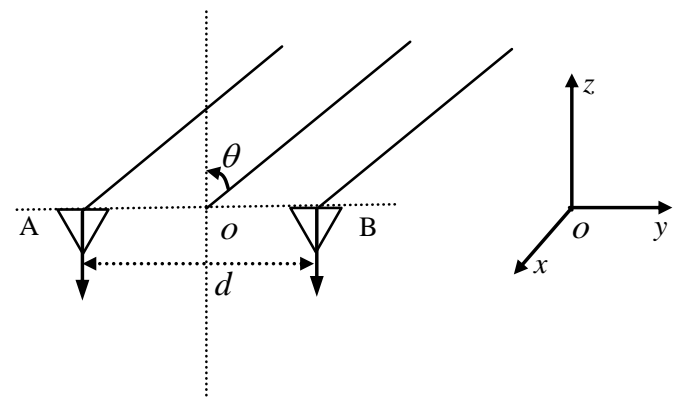

\section{Figure 2. Monopulse Angle Measurement Theorem based on the Conically Conformal Antennas}

\section{Design of the Conical Conformal Monopulse Antenna System based on the LPDA}

The basic design idea of antenna element for passive radar in this paper is to bend the traditional wide band LPDA to make it conformal with the conical antenna radome. The monopulse antenna system consists of two or four conformal LPDA elements mounted on the surface of the conical radar radome, which is used to form the sum and difference patterns for target detection and tracking. Firstly, a traditional planar LPDA was designed and its geometry is shown in Fig.3.The designed LPDA includes 17 dipoles, and a coaxial cable is employed to feed it at the top of the dual transmission line where the shortest dipole is positioned. The length $L$ and the distance $d$ between the adjacent dipoles are decreased along the dipole array by the factor $\tau$, where $\tau$ satisfies the following equation.

$$
\tau=\frac{L_{n+1}}{L_{n}}=\frac{d_{n+1}}{d_{n}}
$$

For the designed geometry in Fig.3, $\tau$ is equal to $0.81, L_{1}$ is equal to $190 \mathrm{~mm}, d_{1}$ is equal to $17 \mathrm{~mm}, H$ is equal to $95 \mathrm{~mm}, H_{1}$ is equal to $5 \mathrm{~mm}, S_{1}$ is equal to $2.65 \mathrm{~mm}$ and $S_{2}$ is equal to 
$0.3 \mathrm{~mm}$. The dipole width $W$ is also decreased along dipole array by the same factor $\tau$ for the numbered dipoles from dipole 1 to dipole 11. Yet, in order to be convenient to fabrication, the numbered dipoles from dipole 12 to dipole 17 have the uniform width which is equal to $0.25 \mathrm{~mm}$. For the designed LPDA, a loaded resistor was used to absorb the reflected waves and ameliorate the voltage standing wave ratio (VSWR) at low frequency. To testify the proposed conformal monopulse antenna scheme, a practical monopulse antenna system with two LPDA elements was designed. The alignment scheme of the two conical conformal LPDA elements is shown in Fig.4. The right hand coordinate system is employed in Fig.4. Fig. 5 shows the structure model of conformal monopulse antenna system with a supporting cone. Here a foam cone was used because of its small effect on the radiation performances of the antenna system. The structure parameters of the cone can be seen in the Fig. 3 and the parameters are: $d_{\mathrm{C}}=168.8 \mathrm{~mm}, D_{\mathrm{C}}=318.5 \mathrm{~mm}$ and $H_{\mathrm{C}}=480 \mathrm{~mm}$. The sum and difference patterns can be achieved on one plane for the two monopulse antenna system with two antenna elements. Let the radiation patterns of two antenna elements $f_{1}(\theta)$ and $f_{2}(\theta)$ at the $x o z$ plane, respectively, then the sum and difference patterns can be achieved by the following formulas.

$$
\begin{aligned}
& \Sigma(\theta)=f_{1}(\theta)+f_{2}(\theta) \\
& \Delta(\theta)=f_{1}(\theta)-f_{2}(\theta)
\end{aligned}
$$

Where $\Sigma(\theta)$ and $\Delta(\theta)$ are the sum and difference patterns, respectively.

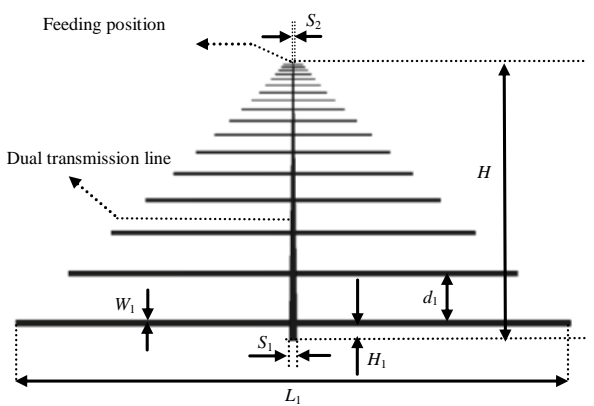

Figure 3. Geometry of the Designed Planar LPDA

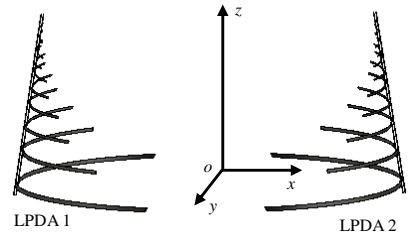

Figure 4. Alignment Scheme of the Two Conical Conformal Monopulse LPDA Elements

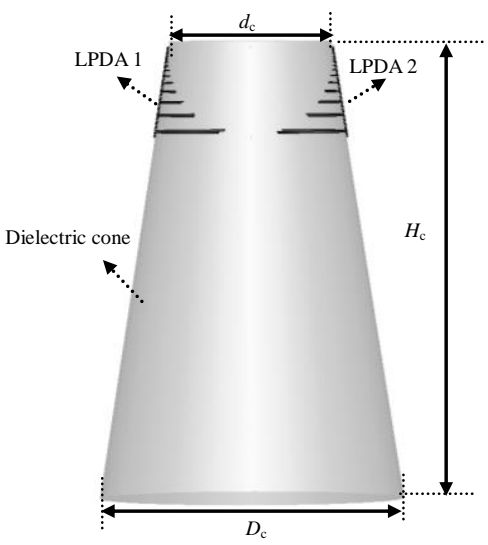

Figure 5. Structure Model of the Conformal Monopulse Antenna System with Supporting Cone 
The designed conformal LPDA monopulse antenna was simulated through full wave electromagnetic simulation software. At the operating plane, the simulated sum and difference patterns are shown in Fig.6 and Fig.7, respectively. Here the simulation results at the frequencies of $1 \mathrm{GHz}, 3 \mathrm{GHz}, 5 \mathrm{GHz}$ and $8 \mathrm{GHz}$ are considered and the corresponding cross polarized patterns are also provided. According to the simulation results, it can be seen that the sum and difference beams are formed. The beam width of radiation pattern becomes narrow when the frequency increases. The cross polarization level of radiation field reduces when the frequency increases. For example, the cross polarization level of sum beam at the bore sight direction at $1 \mathrm{GHz}$ is lower then $-40 \mathrm{~dB}$, yet it is lower than $-25 \mathrm{~dB}$ at $8 \mathrm{GHz}$. The simulation results revealed that the design scheme of the conically conformal LPDA monupulse antenna is feasible.

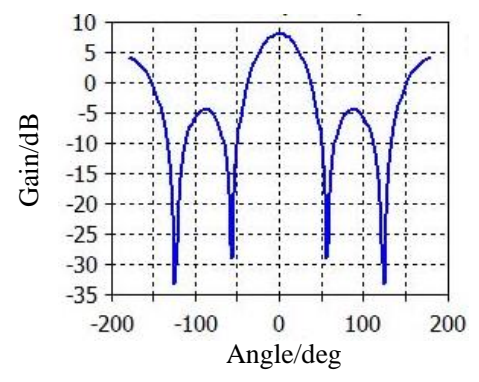

(a)Main Polarization at $1 \mathrm{GHz}$

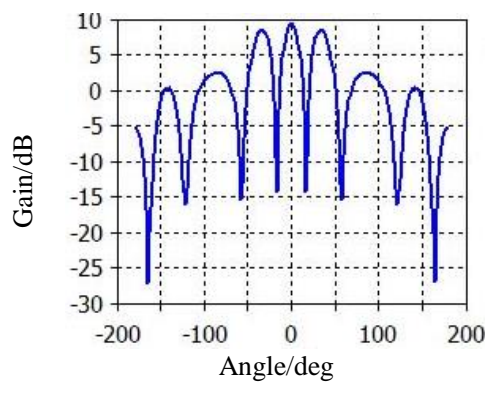

(c)Main Polarization at $3 \mathrm{GHz}$

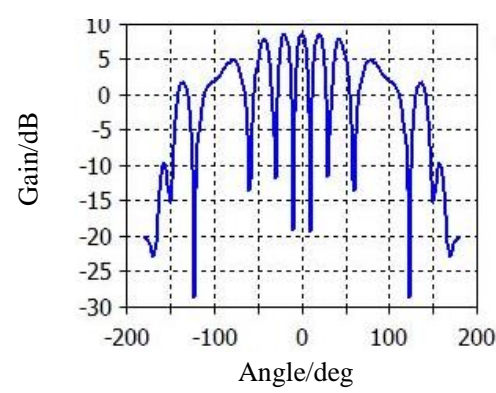

(e)Main Polarization at $5 \mathrm{GHz}$

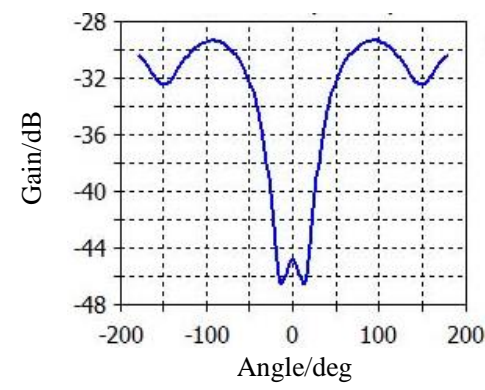

(b)Cross Polarization at $1 \mathrm{GHz}$

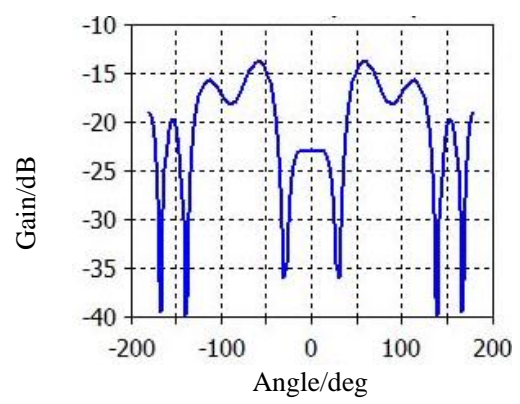

(d)Cross Polarization at $3 \mathrm{GHz}$

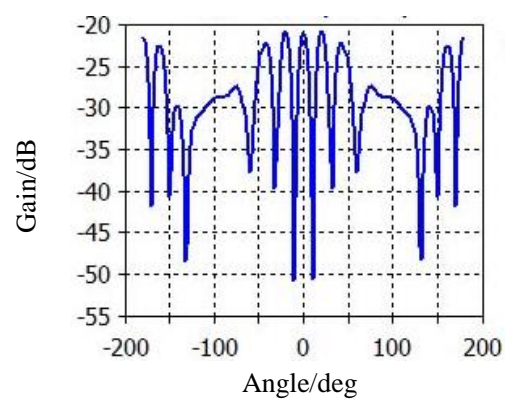

(f)Cross Polarization at $5 \mathrm{GHz}$ 


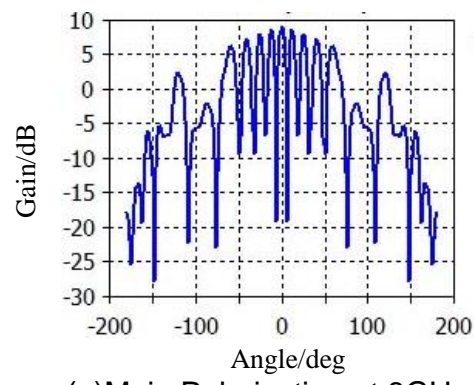

(g)Main Polarization at $8 \mathrm{GHz}$

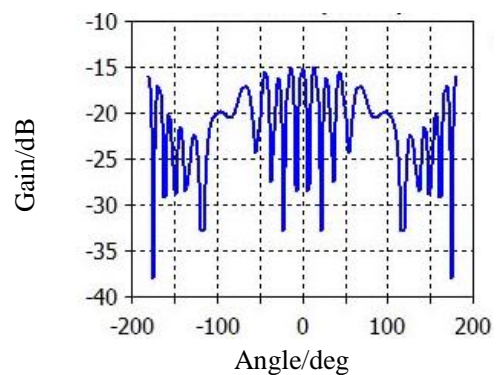

(h)Cross Polarization at $8 \mathrm{GHz}$

Figure 6. The Simulated Sum Patterns of Conically Conformal LPDA Monopulse Antenna

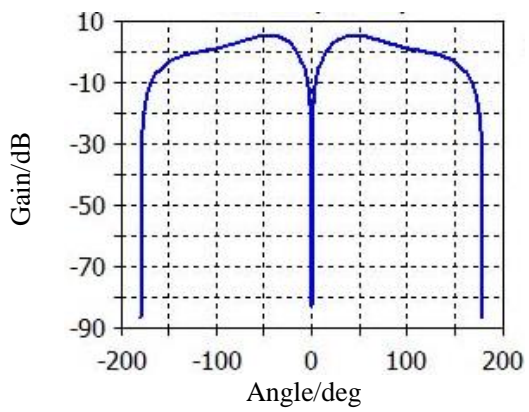

(a)Main Polarization at $1 \mathrm{GHz}$

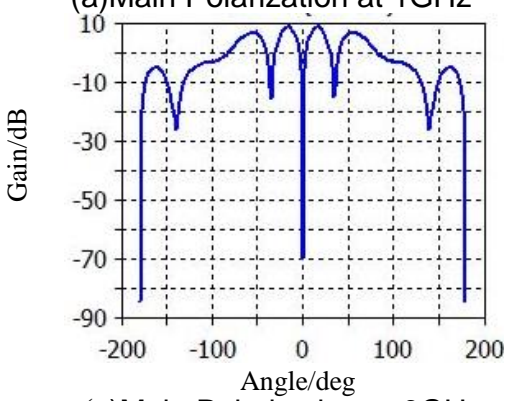

(c)Main Polarization at $3 \mathrm{GHz}$

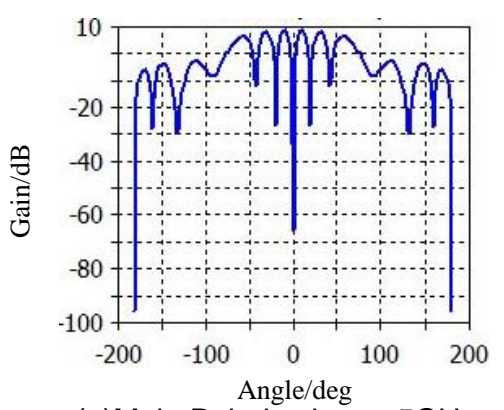

(e)Main Polarization at $5 \mathrm{GHz}$
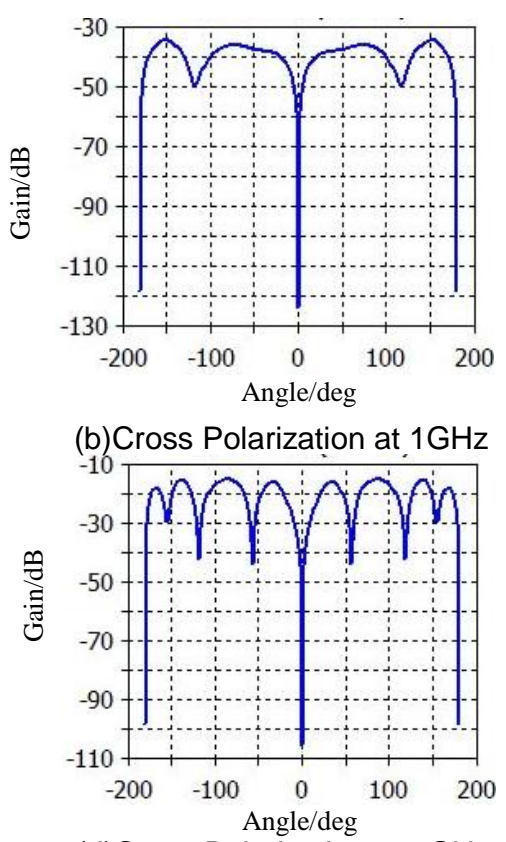

(d)Cross Polarization at $3 \mathrm{GHz}$

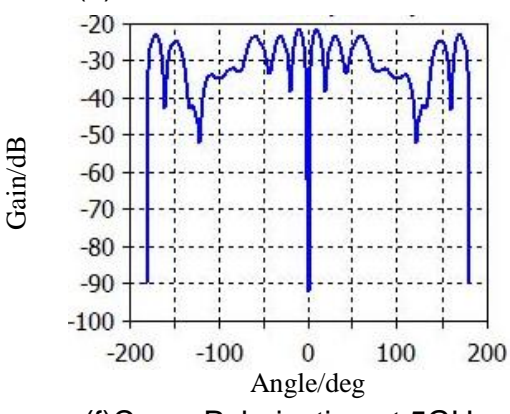

(f)Cross Polarization at $5 \mathrm{GHz}$ 


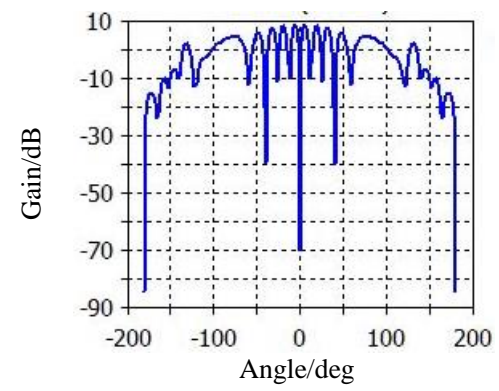

(g)Main Polarization at $8 \mathrm{GHz}$

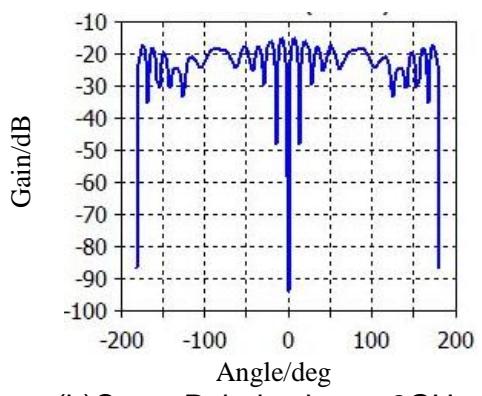

(h)Cross Polarization at $8 \mathrm{GHz}$

\section{Figure 7. The Simulated Difference Patterns of Conically Conformal LPDA Monopulse Antenna}

\section{Experimental Results and Analysis}

A conically conformal monopulse antenna was fabricated and its photograph is shown in Fig.8. The fabricated conformal monopulse antenna was measured in order to obtain its operating performances. The VSWR of each antenna element and isolation degree between two antenna elements were measured by the E8632B vector network analyzer. The measured VSWR of one antenna element and isolation degree between two antenna elements are shown in Fig.9 and Fig.10, respectively. The measured average VSWR is about 2 over the operating frequency range of $1 \mathrm{GHz}$ to $8 \mathrm{GHz}$, which can be acceptable for the wide band electronic systems. The isolation degree is larger than $16 \mathrm{~dB}$ over the operating frequency range of $1 \mathrm{GHz}$ to $8 \mathrm{GHz}$, and when the frequency increases, it becomes larger. Furthermore, the isolation degree is larger than $20 \mathrm{~dB}$ when the frequency is higher than $1.8 \mathrm{GHz}$.

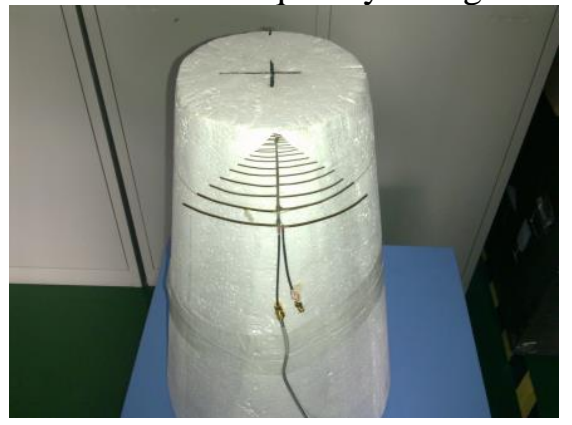

Figure 8. Photograph of the Fabricated Monopulse LPDA

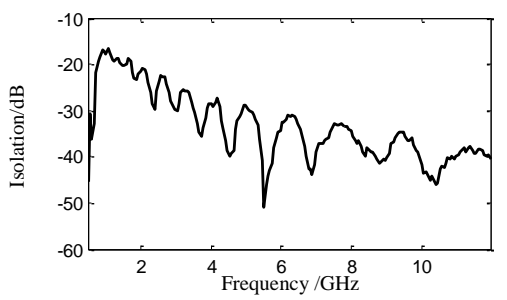

Figure 10. Measured Isolation between Two Antenna Elements

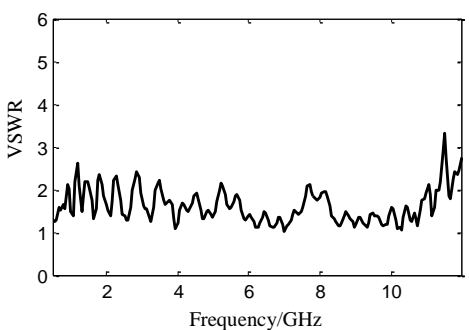

Figure 9. Measured VSWR of the Fabricated LPDA

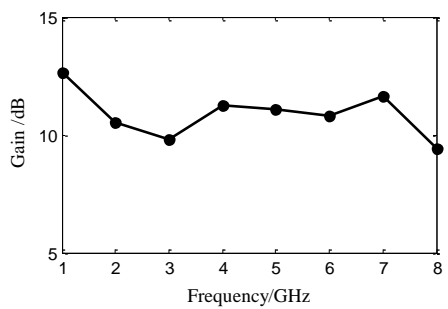

Figure 11. Measured Gain of the Sum Pattern 
In the antenna measurements, the main polarization is defined as the vertical polarization and cross polarization is defined as horizontal polarization at the xoz plane. Fig.12 and Fig.13 show several radiation patterns of LPDA 1 and LPDA 2, respectively. For each antenna element, wide beam and low cross polarization level can be observed, moreover, the cross polarization level increases and the beam becomes narrower when the frequency increases. The measured beam widths of each antenna element at $1 \mathrm{GHz}$ and $8 \mathrm{GHz}$ are about 100 degree and 80 degree, respectively. The cross polarization levels of each element at bore sight for $1 \mathrm{GHz}$ and $8 \mathrm{GHz}$ are lower than $-21 \mathrm{~dB}$ and $16 \mathrm{~dB}$, respectively. The pattern jitter can also be observed for the high frequencies which may be caused by the coupling between two antenna elements or antenna fabrication.

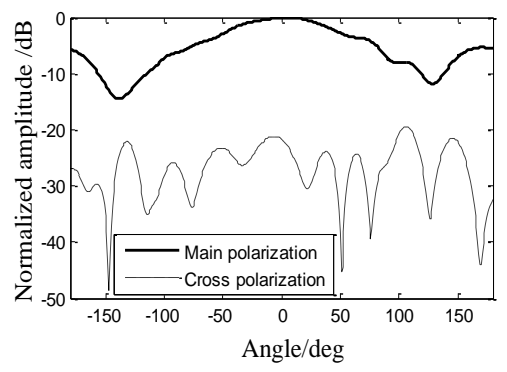

(a) $f=1 \mathrm{GHz}$

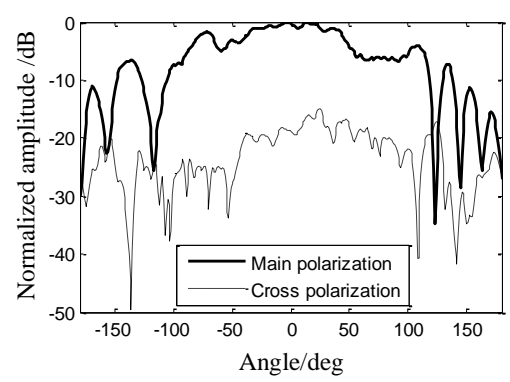

(c) $f=5 \mathrm{GHz}$

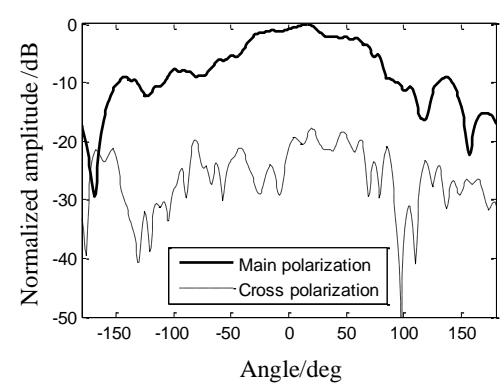

(b) $f=3 \mathrm{GHz}$

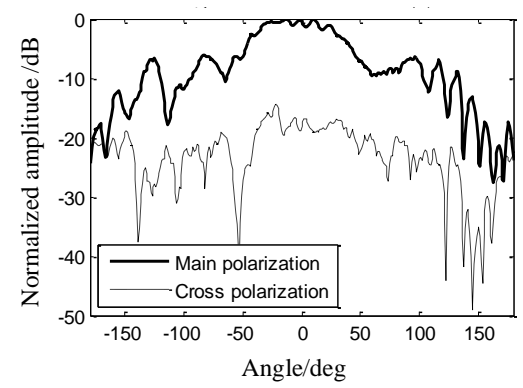

(d) $f=8 \mathrm{GHz}$

Fig.12 Measured patterns of the LPDA 1.

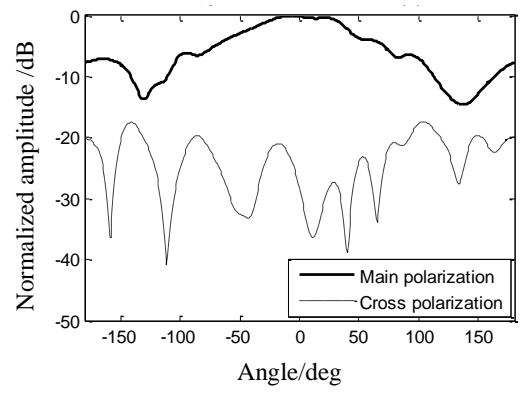

(a) $f=1 \mathrm{GHz}$

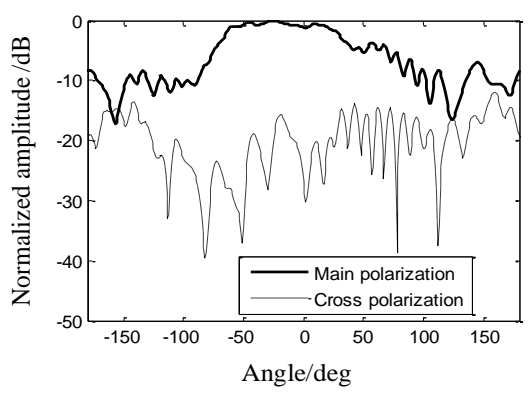

(b) $f=3 \mathrm{GHz}$ 


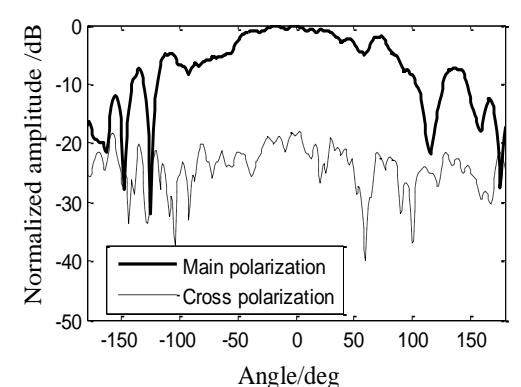

(c) $f=5 \mathrm{GHz}$

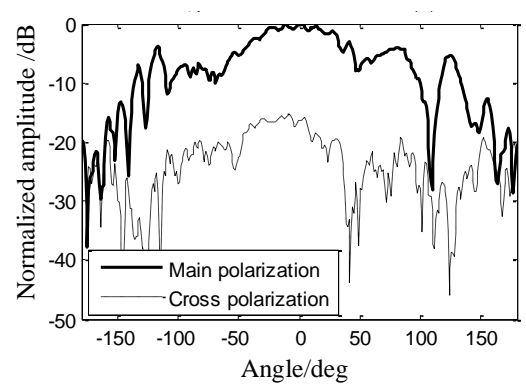

(d) $f=8 \mathrm{GHz}$

Figure 13. Measured Patterns of the LPDA 2

The sum and difference radiation patterns of the fabricated monopulse antenna system were achieved according to the formulas (2) and (3). Here the sum and different patterns of cross polarization are also calculated through independent received data of two antenna elements at cross polarization channels. The measured sum patterns are shown in Fig. 10 and the difference patterns are shown in Fig.11. It can be seen that the sum and difference patterns over the operating frequency range of $1 \mathrm{GHz}$ to $8 \mathrm{GHz}$ were well formed, which demonstrate that the conical monopulse antenna system with wide band based on log-periodic antennas is feasible. The beam width of sum pattern becomes narrower and the null depth of difference becomes lower when the operating frequency increases. The beam widths of sum patterns at $1 \mathrm{GHz}, 3 \mathrm{GHz}, 5 \mathrm{GHz}$ and $8 \mathrm{GHz}$ are about 47 degree, 16 degree, 9 degree and 6 degree, respectively. The null depth of difference patterns at $1 \mathrm{GHz}, 3 \mathrm{GHz}, 5 \mathrm{GHz}$ and $8 \mathrm{GHz}$ are about $-20.2 \mathrm{~dB},-26.6 \mathrm{~dB}$, $29.4 \mathrm{~dB}$ and $-33 \mathrm{~dB}$, respectively. At the same time, it should be noticed that the angle measurement ambiguity appears when the frequency increases, which is caused by the large distance between two antenna elements for high frequency. The problem of angle measurement ambiguity can be solved by employing traditional method used in monopulse radars. Furthermore the polarization characteristics of the radiation patterns for the monopulse antenna systems can be observed in Fig 14 and Fig.15. Just like the polarization characteristic of single LPDA, the increasing trends for the cross polarization level of both the formed sum patterns and formed difference patterns are presented. The cross polarization levels at bore sight of the sum patterns at $1 \mathrm{GHz}$, $3 \mathrm{GHz}, 5 \mathrm{GHz}$ and $8 \mathrm{GHz}$ are about $-25.3 \mathrm{~dB},-22.3 \mathrm{~dB},-19.1 \mathrm{~dB}$ and $-17.7 \mathrm{~dB}$, respectively. The measured polarization characteristics of the monopulse antenna system in this letter demonstrate that conical conformal monopulse antenna for aforementioned operating conditions has approximate linear polarization radiation performances, though the polarization characteristics changes to some extent compared with the planar LPDA. The design scheme of conformal monopulse antenna system for passive radar can also be expanded to a monopulse system with four antenna elements, which can realize the two dimension angle measurements. 


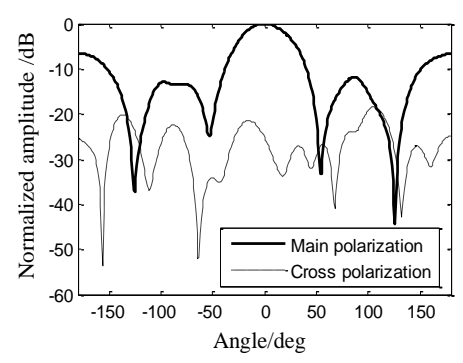

(a) $f=1 \mathrm{GHz}$

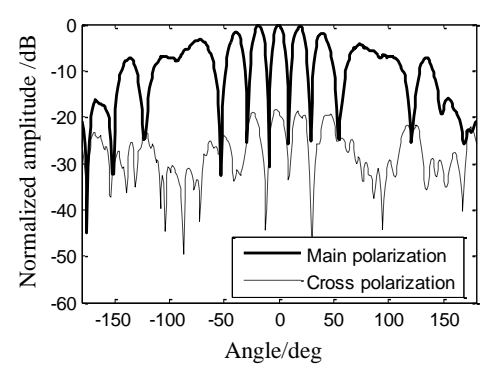

(c) $f=5 \mathrm{GHz}$

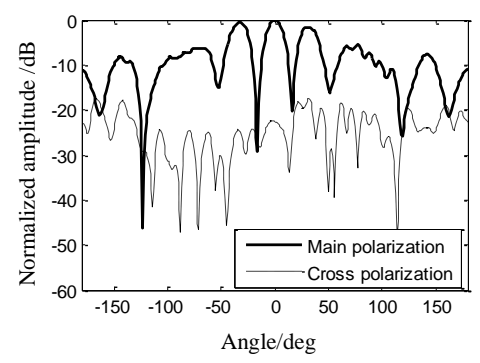

(b) $f=3 \mathrm{GHz}$

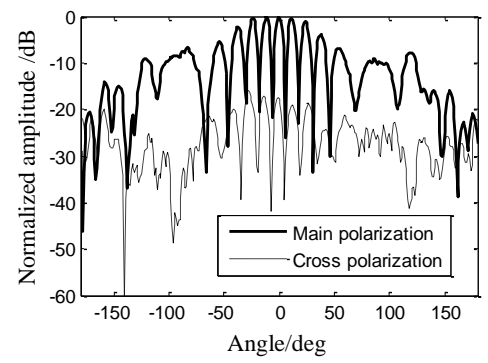

(d) $f=8 \mathrm{GHz}$

\section{Figure 14. Measured Sum Radiation Patterns}

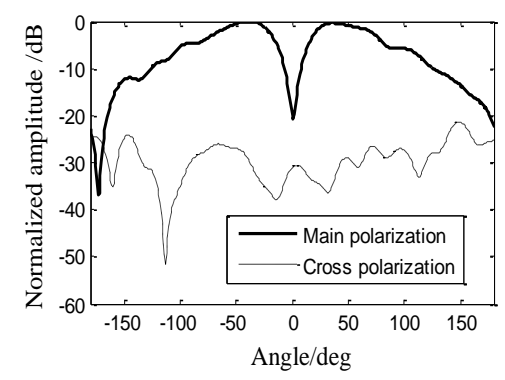

(a) $f=1 \mathrm{GHz}$

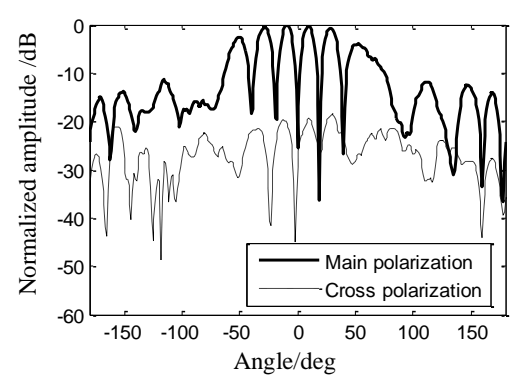

(c) $f=5 \mathrm{GHz}$

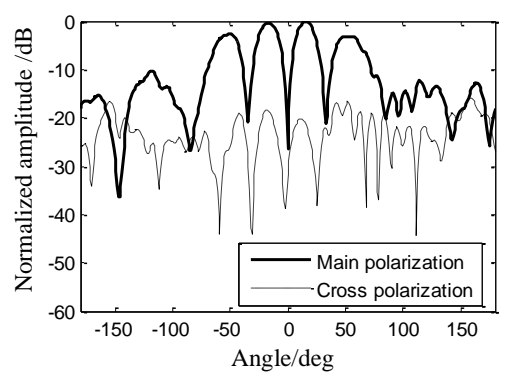

(b) $f=3 \mathrm{GHz}$

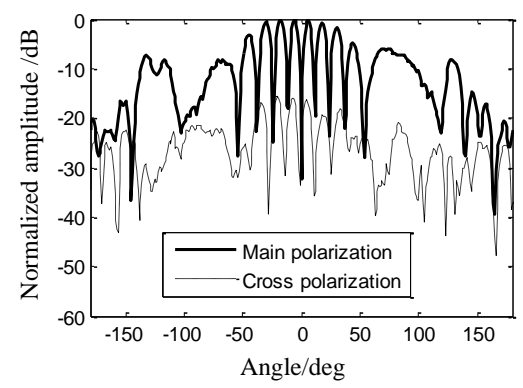

(d) $f=8 \mathrm{GHz}$

\section{Figure 15. Measured Difference Radiation Patterns}

\section{Conclusions}

In this paper, an implementation scheme of wide band monopulse antenna system employing conical conformal $\log$ periodic antennas for the PRS application was 
presented. The proposed antenna system has simple structure and can effectively save the installation space and improve the radiation performances at low frequency, so it is very suitable for the passive radar on the missile. To testify the proposed wide band conformal antenna scheme, a practical monopulse antenna system with two conformal antenna elements was fabricated and the radiation performances are measured and analyzed. The experimental results demonstrate the sum and difference radiation patterns can be formed over the wide frequency range of $1 \mathrm{GHz}$ to $8 \mathrm{GHz}$, so the proposed monopulse antenna system is feasible. What's more, the conically conformal LPDA antenna array can also be used to realize other angle measurement methods such as angle superior resolution technique in wide band passive radar systems.

\section{Acknowledgements}

This work was supported by Project funded by China Postdoctoral Science Foundation (2014M561554) and the Astronautical Supporting Technology Foundation of China (2013-HT-HGD06).

\section{References}

[1] B. Subbarao and V. F. Fusco, "Single Aperture Monopulse Horn Antenna", IEEE Microwave and Wireless Component Letters, vol.15, no.2, (2005), pp.80-82.

[2] P. Rocca, L. Manica and A. Massa, "Hybrid approach for sub-arrayed monopulse antenna synthesis", Electronics Letters, vol.44, no.2, (2008), pp.75 - 76.

[3] G. Cortes-Medellin, "Non-Planar Quasi-Self-Complementary Ultra-Wideband Feed Antenna", IEEE Transactions on Antennas and Propagation, vol.59, no.6, (2011), pp.1935-1944.

[4] N.K. Nahar, B. Raines, R.G. Rojas and B. Strojny, "Wideband antenna array beam steering with free-space optical true-time delay engine", IET Microwaves, Antennas \& Propagation, vol.5, no.6, (2011), pp. 740-746.

[5] Ph. Gonnet, A. Sharaiha, C. Terret and A. Skrivervik, "Feeding Network for Sinuous antennas", Microwave and Optical Technology Letters, vol.20, no.3, (1999), pp.195-200.

[6] M. Vahdani and X. Begaud, "Wideband integrated feeding system for a dual polarisation sinuous antenna", IET Microwaves, Antennas \& Propagation, vol.4, no.11, (2010), pp.1704-1713.

[7] H. Zhou, N. A. Sutton, and D. S. Filipovic, "Surface Micromachined Millimeter-Wave Log-Periodic Dipole Array Antennas", IEEE Transactions on Antennas and Propagation, vol.60, no.10, (2012), pp.4573-4581.

[8] K. Yang, Z. Zhao, Z. Nie, J. Ouyang and Q. H. Liu, "Synthesis of Conformal Phased Arrays With Embedded Element Pattern Decomposition", IEEE Transactions on Antennas and Propagation, vol.59, no.8,(2011), pp.2882-2888.

[9] L. Zou, J. Lasenby and Z. He, "Beamforming with distortionless co-polarisation for conformal arrays based on geometric algebra”, IET Radar, Sonar and Navigation, vol.5, no.8, (2011), pp.842-853.

[10] L. Zou, J. Lasenby and Z. He, "Direction and polarisation estimation using polarized cylindrical conformal arrays”, IET Signal Processing, vol.6, no.5, (2012), pp. 395-403.

[11] M. Costa, A. Richter, and V. Koivunen, "DoA and Polarization Estimation for Arbitrary Array Configurations", IEEE Transactions on Signal Processing, vol. 60, no. 5, (2012), pp.2330-2343.

[12] E. Chaumette, U. Nickel and P. Larzabal, "Detection and Parameter Estimation of Extended Targets using the Generalized Monopulse Estimator", IEEE Transactions on Aerospace and Electronic Systems, vol.48, no.4, (2012), pp.3389-3417.

[13] Y. J. Cheng, W. Hong and K. Wu, "94 GHz Substrate Integrated Monopulse Antenna Array", IEEE transactions on Antennas and Propagation, vol.60, no.1, (2012), pp.121-129.

[14] N. J. G. Fonseca, M. Coudyser, J.-J. Laurin and J.-J. Brault, "On the Design of a Compact Neural NetworkBased DOA Estimation System", IEEE Transactions on Antennas and Propagation, vol.58, no.2, (2010), pp.357-366. 


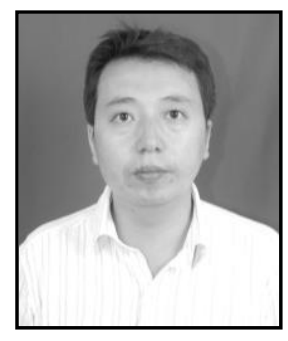

Lizhong Song, he was born in 1975. He received master degree and Ph.D. degree from Harbin Institute of Technology in 2001 and 2005 , respectively. He is a professor and doctoral supervisor of Harbin Institute of Technology at Weihai. Now he is a post doctor of State Key Laboratory of Millimeter Wave in Southeast University. $\mathrm{He}$ focuses his academic interests on antenna design, wireless electromagnetic wave propagation, microwave technology and radar signal processing. 
International Journal of Future Generation Communication and Networking Vol. 8, No. 3 (2015) 